\title{
Apport De La Prospection Géoelectrique (Tomographie Et Trainé Éléctrique) À L'étude Des Tourmalinites Stanniferes d'Achmmache (Maroc Central)
}

\author{
Moussaid Azizi \\ Aissa Mohamed \\ Mezougane Hafid \\ Kharis Abdel-Ali
}

Laboratoire d'Etudes des Ressources Minérales et Energétiques, Département de Géologie, Faculté des Sciences, Zitoune, Meknès, Maroc,

\section{Hritta Driss \\ Boualoul Mustapha}

Laboratoire d'Ingénierie géologique, Département de géologie, Faculté des Sciences.

\begin{abstract}
The Achemmache region is located in the East of El Hammam district, in the Eastern part of the Northern Hercynian Central Morocco; Tin concentrations, economically exploitable, have been recognized in brecciated tourmaline structures of Late Visean, and generally oriented E-W. Therefore, they contrast with other tourmaline oriented NE-SW which proved barren. In order to keep the horizontal and vertical continuity as well as the dip and extension of mineralized structures in the sector, a geophysical survey by Horizontal Electrical resistivity (TE) prospecting and Electrical Tomography was performed in the region. The TE1 profile was performed in the West Zone where the mineralized structures outcrop; it allows highlighting four positive anomalies, while the TE2 profile was carried out in Eastern zone of the sector where the mineralized structures are largely hidden. It can also bring out only two rather distant positive anomalies. These anomalies correspond to mineralized structures of tourmaline. While a duplicate executing of TE1 and TE2, mineralized structures show that some resistant ones have different dips, while some others are subvertical. The TM1 profile was carried out at the same location of electrical TE1 profile in order to confirm the tourmaline structures. The TM1 shows from the NW to the SE an alternation of resistant levels with the relatively conductive levels. The resistive levels are correlated
\end{abstract}


with tourmaline structures identified by the horizontal electrical resistivity. The structures dips show a good correlation between the electrical tomography results and these obtained by the horizontal electrical resistivity profiles with duplicate mode. The study of thin sections and polished sections of the SCA110 and SCA8 core drilling, carried out by BRPM, allowed to recognize, at the mineralized zones, a paragenesis composed of gangue minerals such as quartz, calcite, tourmaline metallic minerals, sulphides and oxides such as magnetite and cassiterite.

Keywords: Achemmache, Tourmaline, geoelectric, electrical tomography, tin, mineralogy

\section{Résume}

La région d'Achmmache est située à l'Est du district minier d'El Hammam dans la partie Nord orientale du Maroc Central Hercynien. Des concentrations d'étain, économiquement exploitables, ont été reconnues dans des structures de tourmalinites bréchiques du viséen supérieur d'orientation générale $\mathbf{E}-\mathbf{W}$. Elles contrastent de ce fait avec d'autres tourmalinites de direction NE-SW qui sont stériles. Les terrains du viséen moyen et supérieur encaissant la minéralisation sont de nature flyschoide. Ils sont discordants sur une série stratigraphique qui débute par des schistes noirs à graptolites du Silurien, surmontés par des schistes gréseux à nodules calcaires du Dévonien. La paragenèse minérale de ces structures est composée essentiellement de quartz, calcite, tourmaline, sulfures (pyrite, chalcopyrite, arsénopyrite..) et oxydes (principalement : cassitérite, magnétite et rutile). Le secteur étudié, au niveau du J. Achmmache, comporte un certain nombre de structures de tourmalinites (I, J, Z1 et $\mathbf{Z 2}$ ), à teneur importante en Sn vers le NE (permis miniers de la société KASBAH) qui ont tendance à affleurer par intermittence vers l'Ouest (permis miniers de SAMINE). Dans le but de suivre la continuité latérale, ainsi que le pendage et le prolongement de ces structures, des essais géophysiques par prospection géoélectrique (deux profils TE1 et TE2 en double de longueur ligne) et tomographie électrique (un profil TEM), ont été réalisés au niveau de ce secteur. Le profil électrique TE1 est effectué dans la zone ouest où affleurent les structures minéralisées. Il a permis de mettre en évidence quatre anomalies positives., alors que Le profil électrique TE2, réalisé plus à l'Est du précédent (200m environ), dans une zone où les structures minéralisées sont en grande partie masquées, n'a permis de mettre en évidence que deux anomalies positives assez éloignées correspondant à la continuité des structures I et J. Les pendages des structures minéralisées, déduits des profils TE1 et TE2 en double de longueur ligne, montrent qu'ils sont globalement redressés, avec des inclinaisons en sens opposés (NW et SE). Ils seraient contrôlés par le plissement hercynien majeur, repris par les 
jeux conjugués des failles locales. Le profil TM1 a été réalisé à l'endroit du profil profil électrique TE1. Il montre du NW vers le SE une alternance de niveaux résistants et de niveaux relativement conducteurs. Les niveaux résistants correspondraient aux structures de tourmalinites décelées par le profil électrique. Les résultats de la tomographie corroborent donc les résultats obtenus par les profils électriques aussi bien au niveau de la continuité latérale qu'au niveau des pendages. L'étude des lames minces et des sections polies provenant de sondages carottés SCA10 et SCA8, effectués par SAMINE, a permis de reconnaître au niveau des zones minéralisées une paragenèse composée de minéraux de gangue tels que le quartz, la calcite et la tourmaline et minéraux métallique, les sulfures, les oxydes tels que la magnétite et cassitérites.

Mots clés : Achmmache, Tourmalinites, Géoéléctrique, Tomographie électrique,Etain, Minéralogie

\section{Introduction}

Le district d'El Hammam est situé au NE du Maroc Central. Il est connu surtout pour sa minéralisation de fluorine, qui est actuellement, exploitée par la société minière SAMINE (groupe ONA). Vers la partie Est se localise le secteur d'Achemmache, caractérisé par la présence des structures de tourmalinite minéralisées en étain ; ces derniers sont contrôlées par des fractures d'orientations variables, avec une prédominance de deux directions principales : E-W et NE-SW.

Dans la partie Ouest d'Achèmmache la plupart de ces structures apparaissent en surface, alors qu'elles sont en grand partie masquées à l'Est du secteur. L'objectif est de suivre leurs continuités horizontales et verticales. C'est dans cette optique qu'on a utilisé deux méthodes géophysiques indirectes, la méthode géoéléctrique par trainés électrique et la tomographie électrique.

\section{SITUATION GEOGRAPHIQUE}

Les coordonnées GPS de la mine d'El Hammam sont N 3333'33', W 5'48'23', alt. $700 \mathrm{~m}$. Les bâtiments sont échelonnés entre 660 et $780 \mathrm{~m}$ d'altitude dans une vallée d'un affluent de l'oued Beth. Le gîte d'Achmmache est à $5 \mathrm{~km}$ à l'est de la mine d'El Hammam. Le district s'étend à l'extrême SE de la feuille au 1/50 000 de Bouqachmir, entre les méridiens $5^{\circ} 45^{\prime}$ et $5^{\circ} 55^{\prime}$ et les parallèles $33^{\circ} 30^{\prime}$ et $33^{\circ} 35^{\prime}$. 


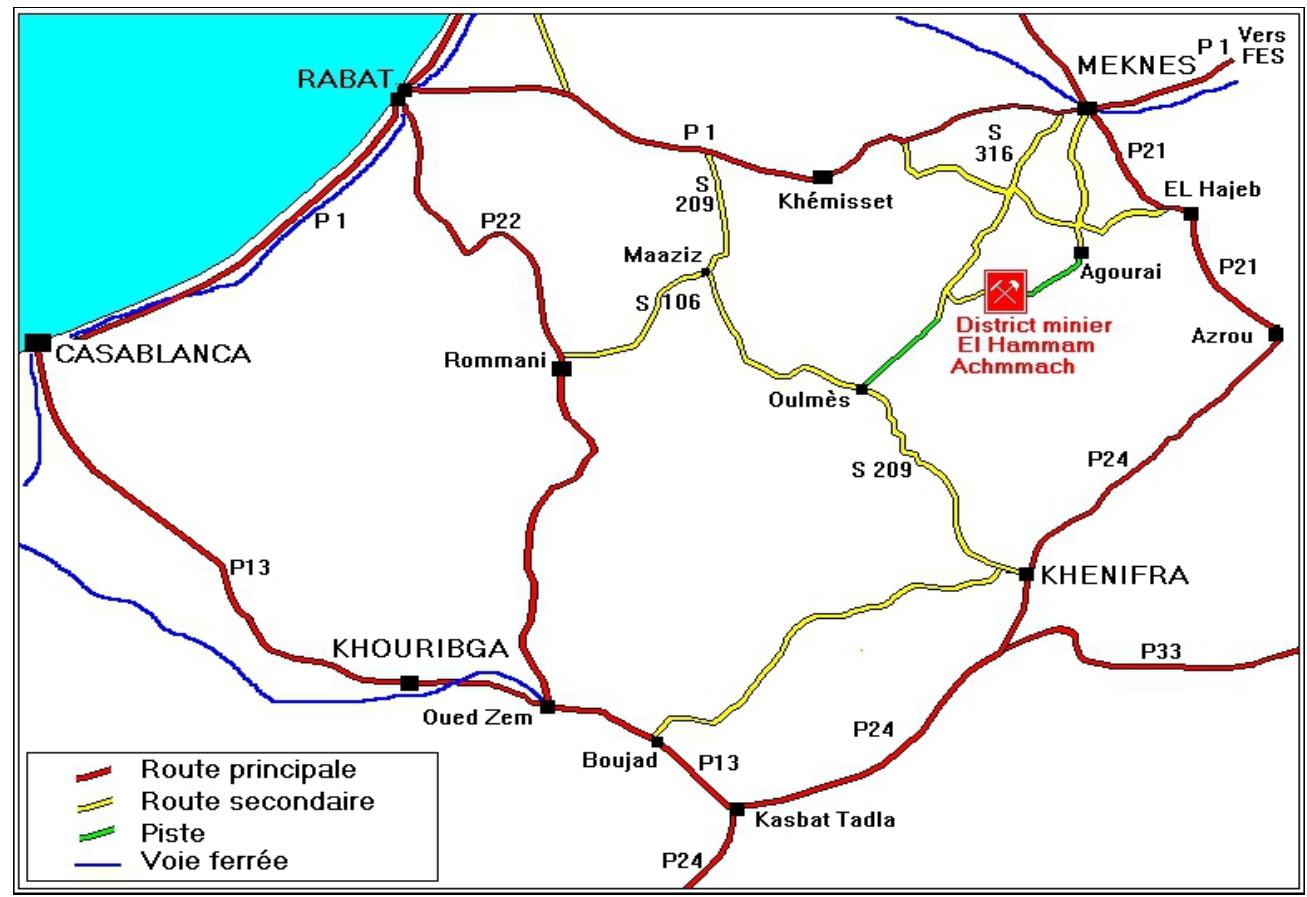

Figure 1 : Localisation du district d'El Hammam-Achmmache

\section{Cadre geologique}

Le Maroc Central Hercynien est la principale unité de la méséta occidentale. Il est constitué d'une succession d'Anticlinorium et de synclinorium séparés par des zones de dislocation qui ont joué en cisaillement lors de la compression tardi-hercynienne et en limites du bassin carbonifère (Michard 1976, Piqué 1980).

Le district dEl Hammam qui se trouve dans la bordure Nord-Est du Maroc Central Hercynien à $60 \mathrm{~km}$ au SW de Meknès, comporte une série stratigraphique qui débute par des schistes noirs à graptolites du Silurien, associés à des quartzites affleurant au NE d'Ain El Hammam. Le Dévonien est représenté par des schistes gréseux à nodules calcaires. Les terrains du viseen moyen et supérieur se déposent en discordance sur les deux séries précédentes (Jebrak et al. 1984). Ils comprennent une série de base schistogérseuse et microconglomératique et une série schisto-calcaire surmontée parfois par des schistes verts et des schistes gris ardoisiers recouverts par les formations rouges du Permo-Trias (Lésier et Yajima 1970). 


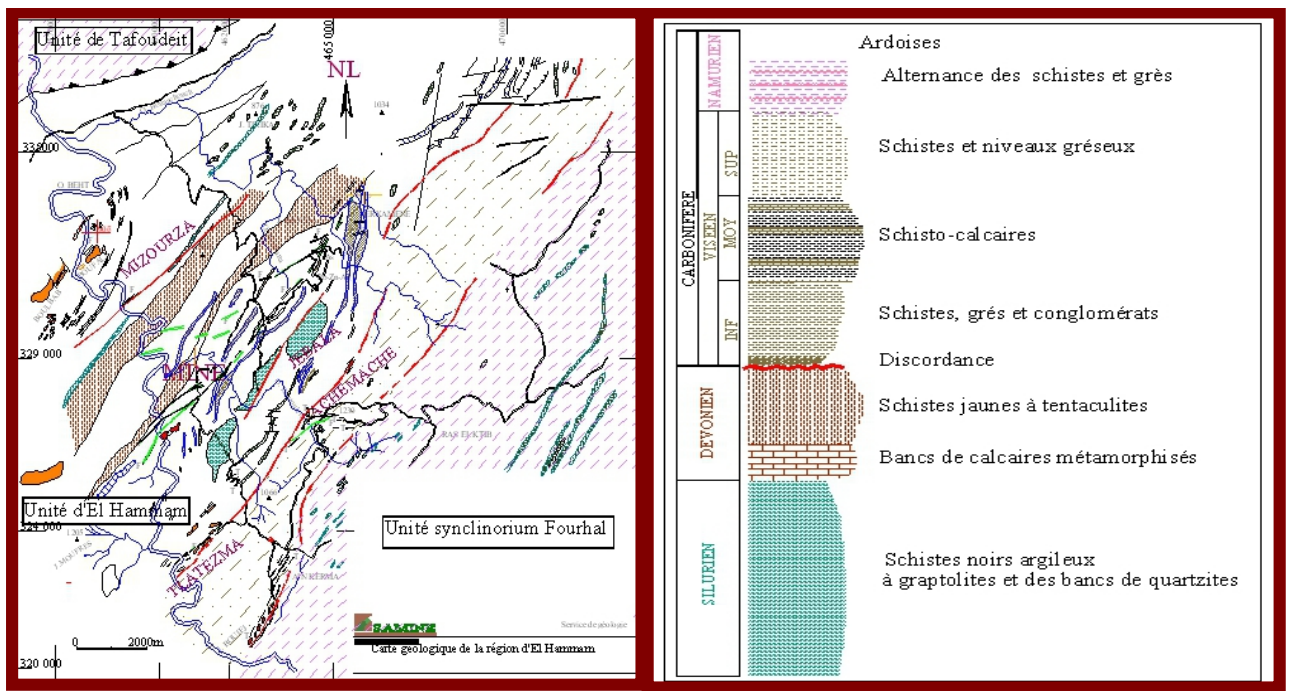

Fig. 2 : carte géologique de la zone d'étude, (Document interne SAMINE)

L'ensemble des formations viseennes est interprétés sur le plan paléogéographique comme une série continue, débutant par des faciès détritiques, puis de plateforme carbonatée de type interne et s'achevant par des sédiments pélitiques de talus. Il s'agirait donc du comblement d'un sillon à subsidence rapide en présence d'une activité tectonique continue (Jebrak 1984). Cette dernière s'est traduite par des phases de plissements post-viséen ayant engendrant une succession de synclinaux et d'anticlinaux orientés NESW et déversés SE (Agard 1966).

La série stratigraphique du district d'El Hammam et du secteur d'Achmmèche est affectée, en plus du métamorphisme régional de type épizonal, par un métamorphisme de contact matérialisé sur le terrain par des schistes tachetés à andalousite et cordiérite. Ce métamorphisme est attribué principalement à la mise en place d'un batholite granitique enfoui connu sous le nom de granite d'Achmmèche et dont l'apophyse d'Oued Beht en constituerait le seul affleurement connu en surface.

La série viseenne de ce district est également recoupée par des dykes d'autres roches magmatiques.

Un examen rapide des roches magmatiques connues dans le district d'El Hammam permet d'en distinguer trois variétés (1) les microgranites et rhyolites, (2) les dolérites et les microdiorites quartziques et (3) le granite de l'oued Beht. L'association de roches magmatiques basiques et acides constitue une constante régionale à l'échelle du Maroc Central. A El Hammam les dolérites seraient antérieures à la mise en place du granite et les microgranites riches en bore et étain sont plus tardifs. Des rhyolites blanches à taches de tourmalines sont recoupées par des microdiorites quartziques à Boujjaj (partie SE du district). 
Le granite d'El Hammam (ou granite de l'oued Beht) constitue la principale manifestation plutonique du district. Il est intrusif dans un encaissant schisteux tacheté du viséen supérieur. Le faciès principal correspond à une roche à grain fin de composition monzonitique péralumineuse, composée de quartz précoce, de feldspath potassique en cristaux xénomorphes à inclusions de plagioclase et biotite, de plagioclase, de biotite, plus ou moins chloritisée et de minéraux accessoires (zircons, apatite, opaques...). Outre le faciès principal, on distingue un faciès tacheté à cordiérite et un faciès apical correspondant à une roche plus ou moins altérée de couleur jaune rouille caractérisant le sommet du granite (muscovitisation des feldspaths, chloritisation des biotites et oxydation de l'ilménite). Ce dernier faciès est à mettre en parallèle avec le faciès greisénisé observé dans les carottes de sondages effectués dans l'oued Beht. En affleurement la gréisénisation est limitée au voisinage de veines à andalousite, muscovite ou de filons à quartz, tourmaline, mispickel et traces de métaux $(\mathrm{Au}, \mathrm{Cu}, \mathrm{Sn}, \mathrm{Bi})$.

Le granite d'El Hammam présente plusieurs caractères pétrographiques et géochimiques qui en font un granite potentiellement stannigène:

- $\quad$ Son origine crustale (granite de type $\mathrm{S}$ ) : granite riche en $\mathrm{Si}$ et peralumineux (Sonnet 1989).

- Son caractère initialement réduit (présence de l'ilménite, absence de magnétite, biotite ferrifère riche en titane).

- Sa teneur non négligeable en étain, notamment dans les faciès transformés (16ppm pour le faciès sain contre 130 pour le faciès greisénisé).

\section{Gisement d'Achemmach}

Il contient une zone de structures stannifères s'étendant sur environ 8 $\mathrm{km}$ en direction NNE. La zone prospectée par le BRPM dans les années 8090 , couvrant environ $4 \mathrm{~km} 2$, a montré l'existence d'un système dominant de filons $\mathrm{N} 70^{\circ} \mathrm{E}$ à pendage nord, combiné à un système de filons $\mathrm{N} 30^{\circ} \mathrm{E}$ à pendage ouest (Barodi \& Chbihi, 1998). Ces « filons » sont remplis d'une brèche à éléments pris à 1'encaissant (grès et pélites), généralement étirés et boudinés, cimentés par une gangue à quartz-tourmaline. La coupe dressée grâce au creusement d'un puits de $85 \mathrm{~m}$ et d'un travers-banc montre une géométrie des structures bréchiques remarquablement planaires, ce qui suggère qu'il s'agit de failles ductiles-fragiles.

Une donnée gîtologique importante est la présence à faible profondeur d'un pluton granitique (probablement la continuation de celui d'El Hammam), bien démontrée par les études de gravimétrie et de résistivité électrique (Barodi \& Chbihi, 1998). 


\section{Mineralogie des filons mineralises}

L'étude de lames minces et de sections polies provenant soit d'affleurements et de tranchées de la partie Ouest de J. Achmmèche soit de sondages carotté effectués par SAMINE, a permis de reconnaître au niveau des zones minéralisées en étain les minéraux suivants

\section{Minéraux de gangue}

Les observations macro et microscopiques des lames minces ont montré que la gangue de la minéralisation est composée essentiellement de quartz, de tourmaline et de carbonates.

Quartz : la silicification qui accompagne la tourmalinisation de la roche encaissante se traduit par l'expression d'un quartz remplissant les espaces intergranulaires de la roche. Elle se développe également en veines de quartz remplissant des fractures affectant la roche gréso pélitique.

Tourmaline : présente de manière abondante aussi bien au niveau de la roche encaissante que dans les veines minéralisées. La tourmaline se présente, selon les plans de coupe, soit en fines aiguilles aciculaires, soit en cristaux plus grossiers à habitus trapus et zonés.

Calcite: elle se présente soit en remplissage des espaces intergranulaires de la roche soit en plages xénomorphes résiduelles résultant vraisemblablement d'une décarbonatation d'anciennes veines à calcite.

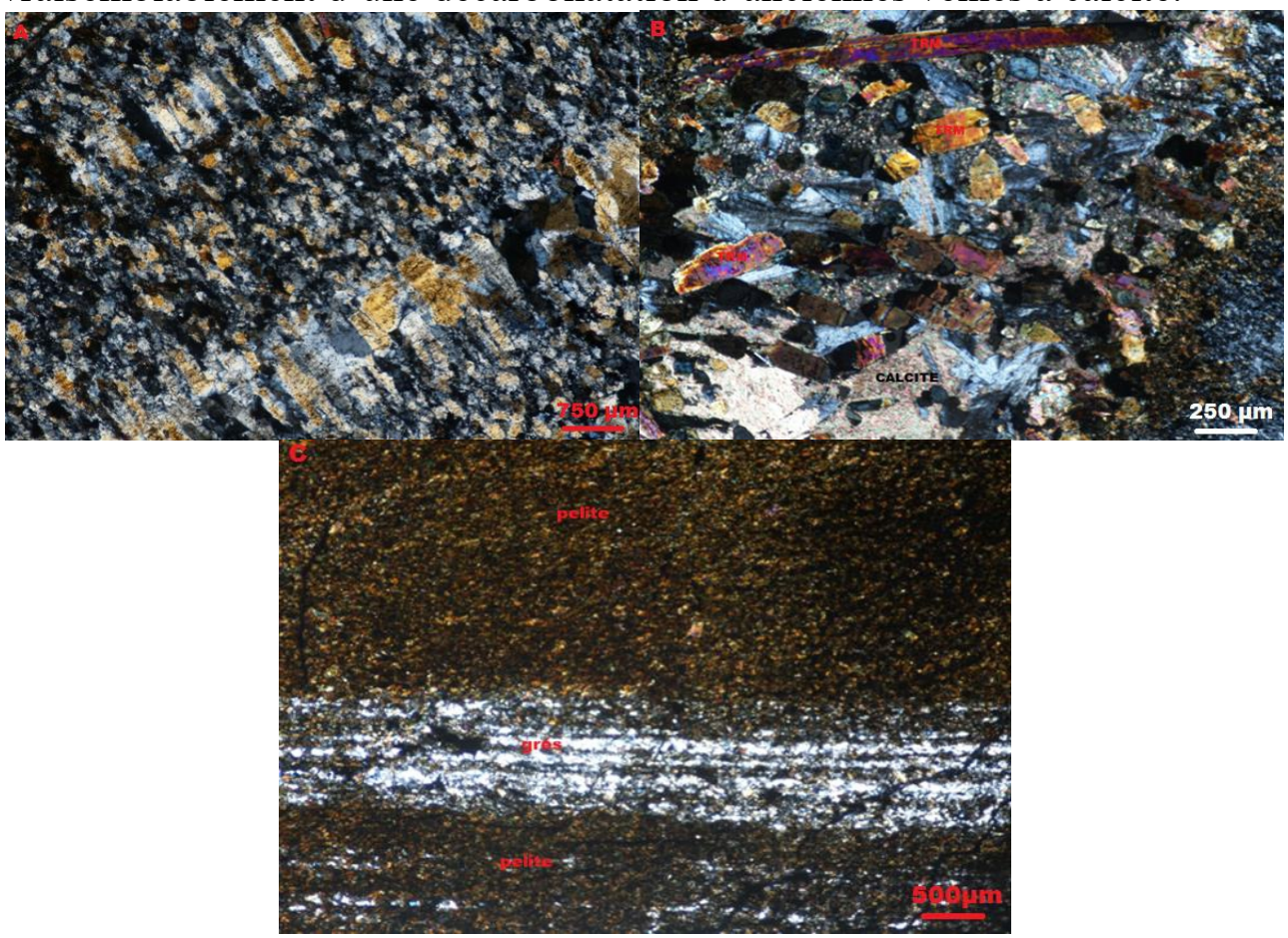

Fig. 3: Photos montrant l'aspect de l'encaissant gréso-pélitique (C) et des veines de quartz (A) et de tourmaline-calcite affectant la roche encaissante (B). 


\section{Minéraux métalliques}

Les minéraux métalliques reconnus dans cette zone d'étude, s'expriment sous forme de sulfures $(\mathrm{Cu}, \mathrm{Fe} . .$.$) et d'oxydes ( \mathrm{Sn}, \mathrm{Ti}, \mathrm{Fe} . .$.$) .$

Chalcopyrite: elle se présente en plages d'ordre $\mu \mathrm{m}$ à $\mathrm{mm}$. Elle est souvent associée à la pyrite et peut montrer des inclusions de cassitérite en cristaux globuleux plus ou moins automorphes. (Photo A).

La pyrite : présente en plages plus ou moins rubanées, associée ou non à d'autres sulfures tels que la chalcopyrite, ou en grains disséminés dans la roche encaissante surtout dans les pélites. Elle peut être aussi présente en veinules monominérales tardives recoupant la roche. (Photo A).

Arsénopyrite : présent, de manière similaire que la pyrite, en cristaux disséminés dans l'encaissant pélitique, ou en plages et agrégats dans les structures filoniennes. (Photo B)

La magnétite et le rutile: Ces oxydes sont présents en grains dissémination, de manière plus ou moins abondante, dans la roche encaissante. (PhotoD)
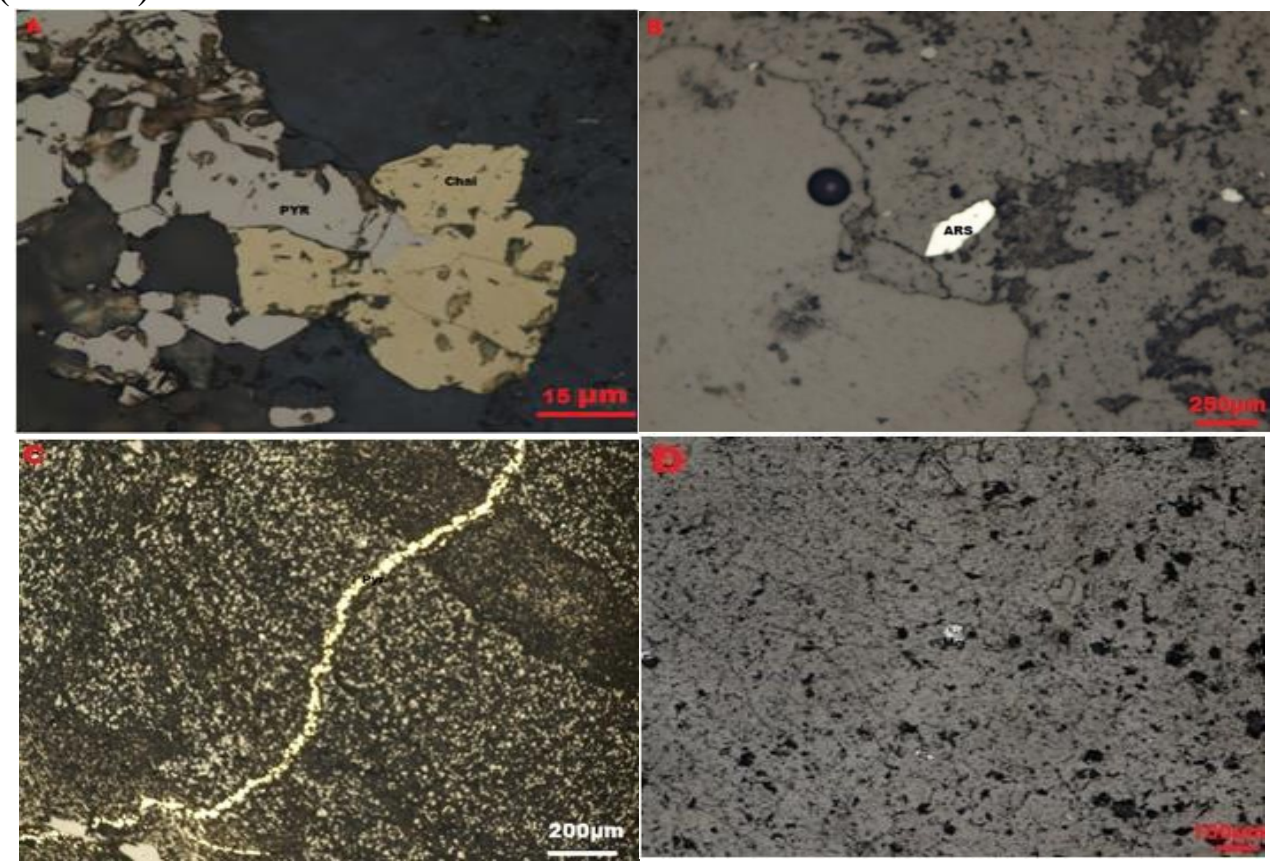

Fig. 4 : Photos montrant des sulfures : Pyrite et chalcopyrite, (A), arsénopyrite(B), Magnetite (B). 


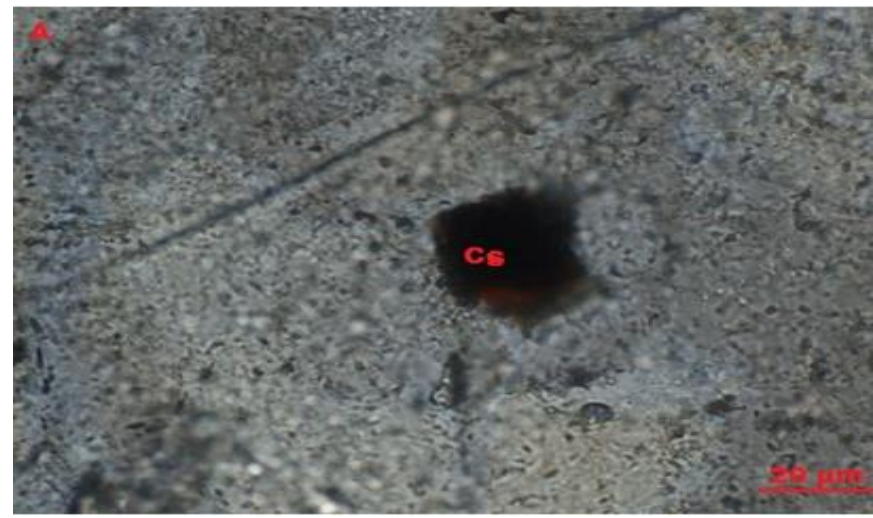

Fig.5 : Photo montrant l'oxyde (Sn) : cassitérite (A).

La Cassitérite : la cassitérite a été reconnue dans quelques lames. Elle est présente sous forme de cristaux globuleux automorphes de petite taille, de manière isolée dans l'encaissant gréso-pélitique ou associées aux sulfures décrits ci-dessus. (Photo A).

Etude géophysique des structures minéralisées au niveau de district d'Achemmache.

Dans le but de suivre la continuité dans les sens horizontal et vertical des structures minéralisées dans le secteur d'Achemmache une campagne géophysique par prospection géoélectrique et tomographie électrique a été réalisée dans la région.

\section{Présentation de méthodes géophysiques utilisées :}

a- méthode géoelectrique :

Principe: La méthode électrique est basée sur la mesure en surface de l'intensité et de la différence de potentiel existante entre les différentes électrodes du dispositif de mesure. Le rapport de ces deux paramètres permet de calculer la résistivité des terrains sous-jacents.
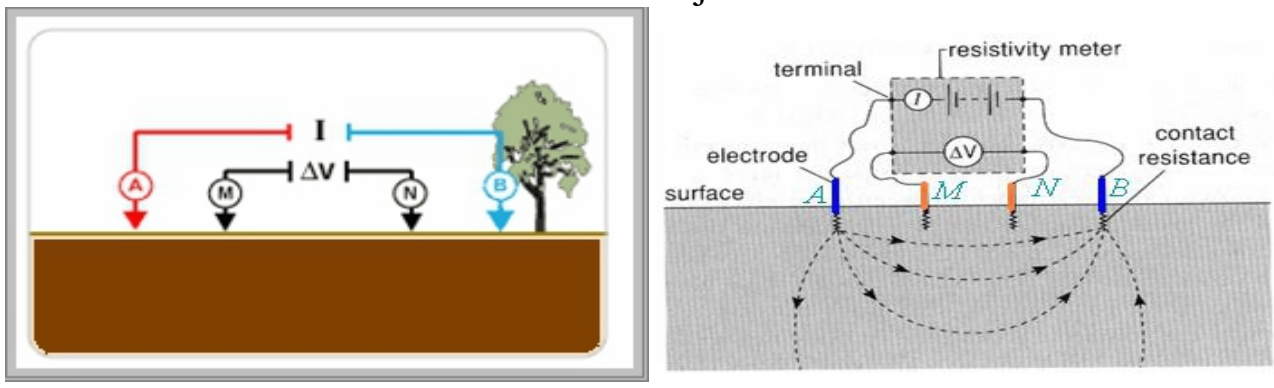

Fig. 6 : Principe de la méthode géo-électrique.

On note la présence de deux techniques fondamentales au sein de la géoélectrique quel que soit le dispositif utilisé, s'agit de technique de sondage électrique et technique de traîné électrique. 
La technique utilisé dans le cadre de ce travail est celle des trainés électriques. Il s'agit de trainer (déplacer) un quadripôle AMNB de dimension fixe et à mesurer la résistivité apparente $\rho_{a}$ en fonction de la position du dispositif ce qui permet une investigation latérale.

L'écartement entre les électrodes étant fixe, la profondeur d'investigation est relativement constante et la résistivité apparente $\rho_{a}$ mesurée dépend des variations latérales des résistivités rencontrées

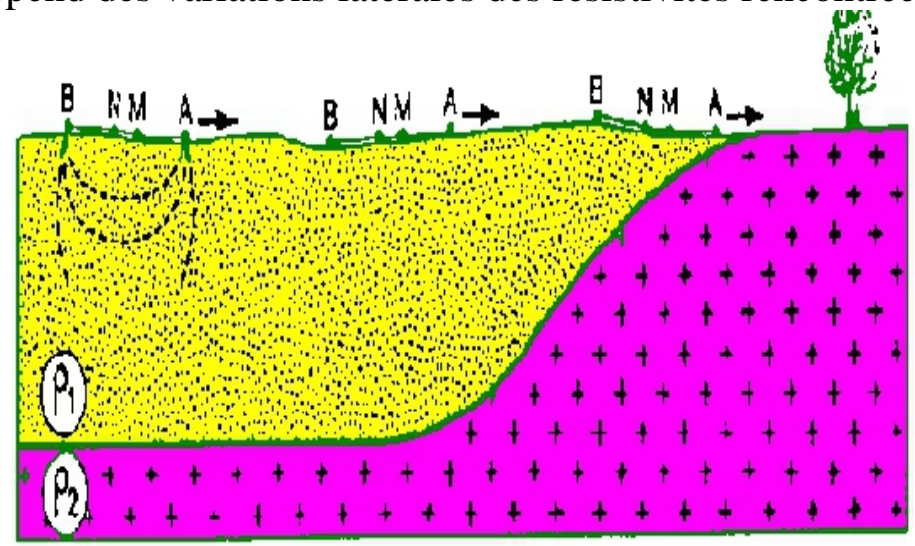

Fig. 7 : Principe des traînés électriques.

Le matériel utilisé est un résistivimètre GéoTrade Mod GTR-3 à inversion de polarité et sommation de signaux avec et un convertisseur générateur de courant 600V de GéoTrade, le dispositif utiliser est Schlumberger.

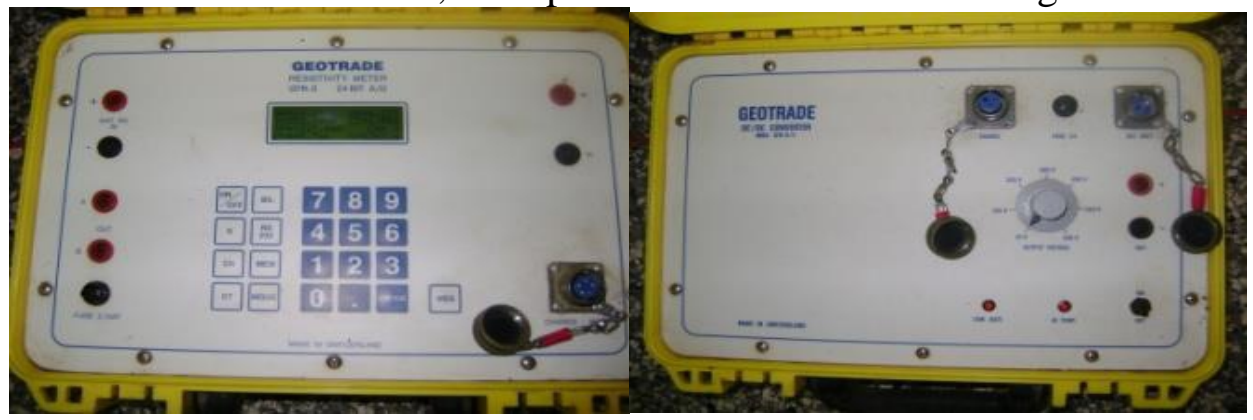

Fig 8 :résistivimètre GéoTrade Mod GTR-3 et convertisseur générateur de courant $600 \mathrm{~V}$ de GéoTrade

b-méthode de tomographie électrique :

Principe : La méthode d'imagerie électrique 2D (tomographie) a été mise au point dans le but d'obtenir un modèle du sous-sol où la répartition de la résistivité varie verticalement et horizontalement le long d'un profil. Dans ce cas, on suppose que la résistivité ne change pas dans la direction perpendiculaire au profil. 
Une acquisition 2D utilise en général un grand nombre d'électrodes connectées à un câble multi-conducteurs et placées selon un profil. Un ordinateur portable, dans lequel est programmée la séquence de mesure, est relié à une boite de commutation et sélectionne automatiquement les électrodes utilisées pour l'injection du courant et la mesure du potentiel (Fig. 9).

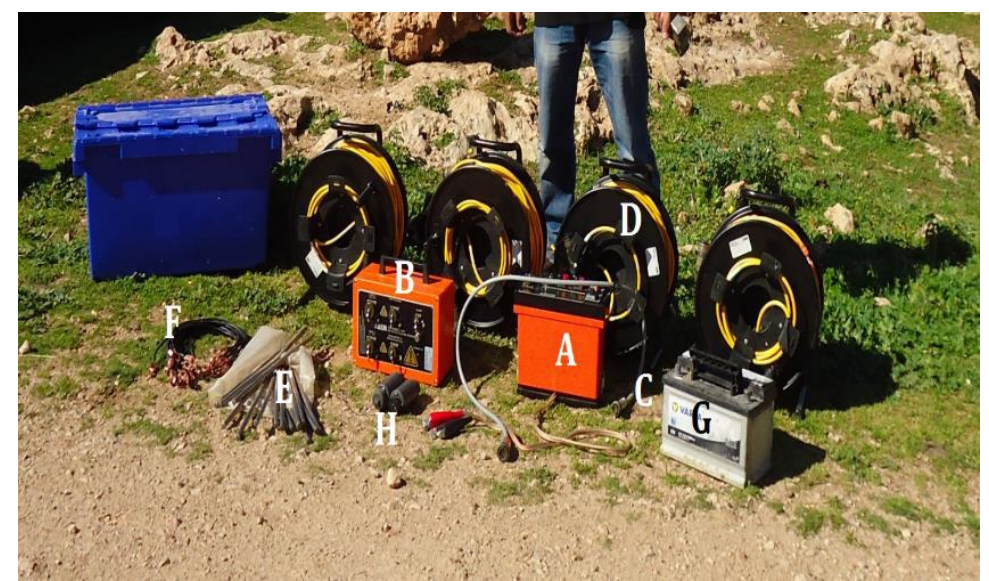

Fig. 9 : photo montrant les instruments d'appareil ABEM de la tomographie électrique.

\section{A) Terrameter 4000; B) Multiélectrode Lund; C) Connecteur appareils; D) Bobine de cable de $160 \mathrm{~m}$; E) Electrodes, F) Connecteurs électrodes- cables, G) Batterie VARTA et $\mathrm{H}$ ) connecteur de cable \\ $>$ Mesures réalisées.}

- 02 profils de traînés électriques en double de longueur de ligne avec des distances AB d'injection de courant de $\mathbf{2 0 0}$ et $\mathbf{3 0 0 m}$.

- 01 profil de tomographie électrique. 


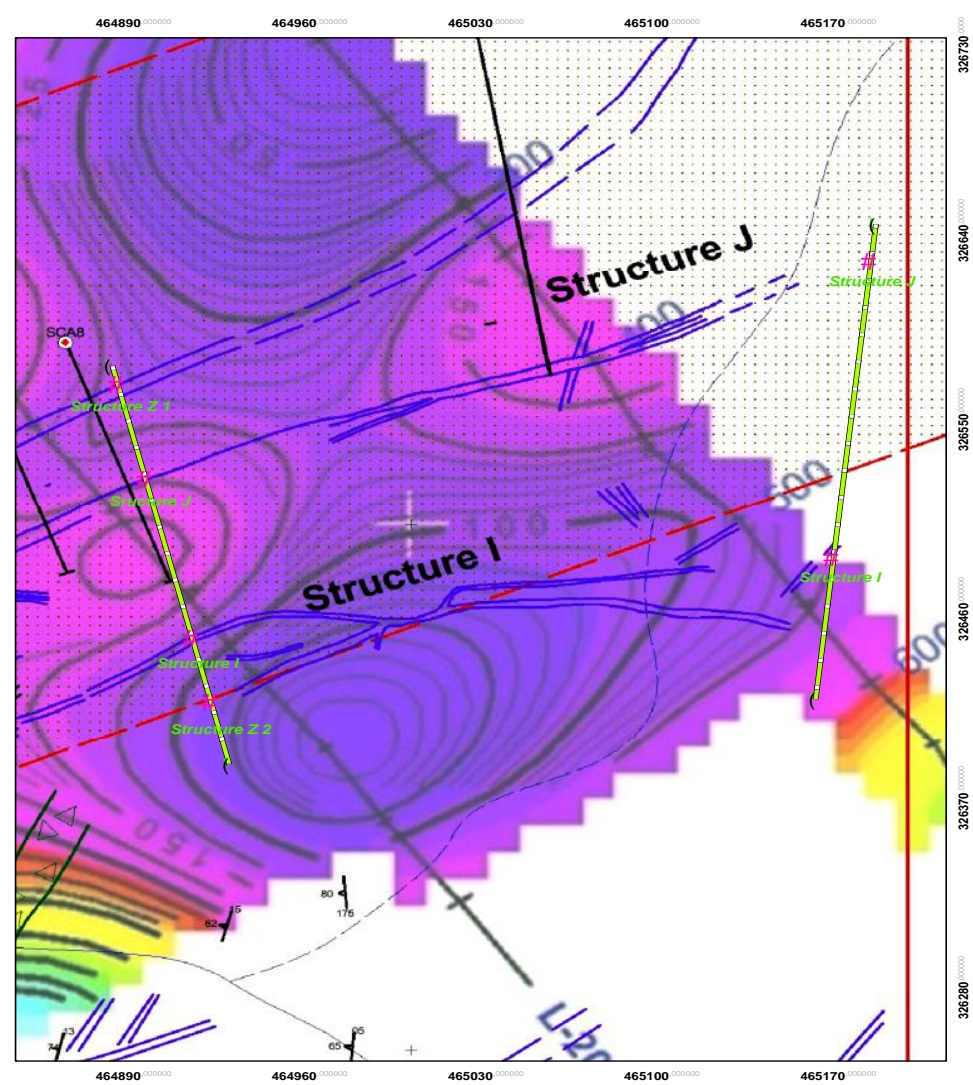

Fig. 10: Implantation des coordonnées des trainées électriques et tomographie électrique sur la carte structurale du secteur.

\section{Résultat des traînés électriques.}

Dans la zone étudiée, deux profils de traînés électriques (TE1 et TE2) ont été exécutés avec une longueur de ligne d'injection de courant $\mathbf{A B}$ de $300 \mathrm{~m}$ correspondant à une profondeur d'investigation d'environ $60 \mathrm{~m}$.

Le trainé électrique TE1 est effectué dans la zone ouest où affleurent les structures minéralisées afin d'obtenir la réponse électrique de ces structures, ceci permettra de faire une corrélation géophysique avec les structures masquées. Le profil TE1 est réalisé selon une direction N160 sur une altitude de $916 \mathrm{~m}$.

Le profil obtenu montre que les résistivités apparentes varient entre 80 et 250 ohm.m. Les structures minéralisées enregistrent des anomalies

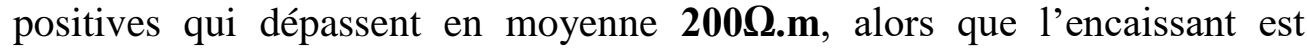
relativement conducteur avec des résistivités qui varient entre $\mathbf{8 0}$ et $\mathbf{1 5 0}$ ohm.m. Quatre anomalies positives A1, A2, A3, et A4 ont été ainsi mis en évidence. (Figure 11) 


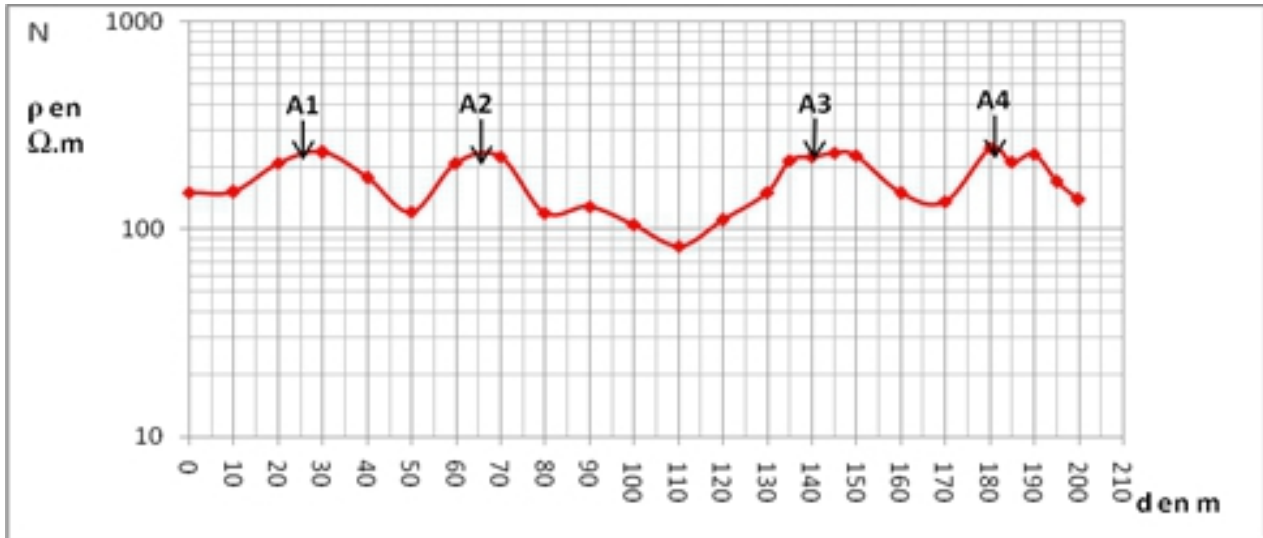

Figure 11 : Profil du traîné électrique TE1 réalisé

La superposition des anomalies sur la carte coïncident avec les structures cartographiées, la première anomalie $\mathbf{A 1}$ correspond à la structure $\mathbf{Z 1}$, la deuxième anomalie $\mathbf{A 2}$ coïncide avec la structure $\boldsymbol{J}$, la troisième $\mathbf{A 3}$ coïncide avec la structure $\boldsymbol{I}$ et la dernière $\mathbf{A} \mathbf{4}$ correspond à la structure $\mathbf{Z 2}$.

Le trainé électrique TE2 est réalisé dans la zone Est du secteur où les structures minéralisées sont en grande partie masquées afin de suivre leur continuité latéralement de l'Ouest vers l'Est.

Le profil TE2 est réalisé avec la même ligne $\mathbf{A B}$ d'injection de courant électrique que le profil TE1 $(\mathbf{3 0 0} \mathbf{~ m})$, sa direction est $\mathbf{N}-\mathbf{S}$, la réponse est donnée dans la figure 12.

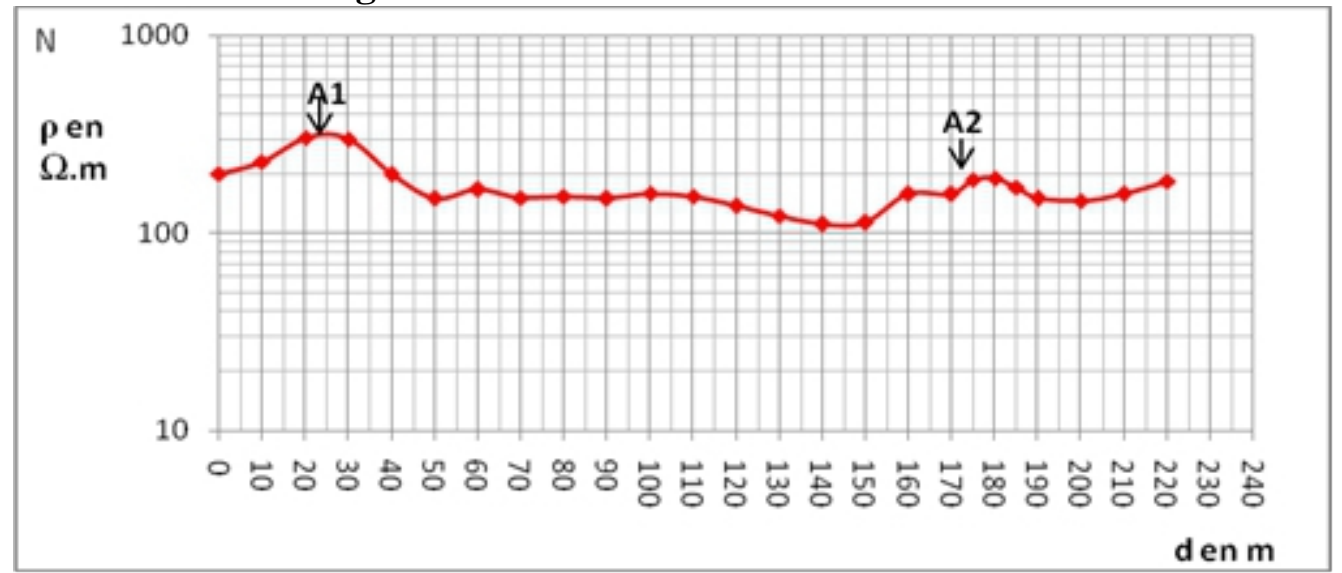

Figure 12 : Profil du TE2.

L'analyse du profil TE2 obtenu permet de montrer que les valeurs de résistivité varient entre $\mathbf{1 0 0}$ et $\mathbf{3 0 0} \mathrm{ohm} . \mathrm{m}$ comme valeur maximale ; il permet de mettre en évidence aussi seulement deux anomalies positives (A1 et A2) assez éloignées. Ceci est expliqué par le fait que les quatre structures minéralisées ne sont pas continues dans l'espace. En effet, la superposition de 
ces anomalies sur la carte montre qu'elles coïncident probablement avec les structures $\boldsymbol{J}$ et $\boldsymbol{I}$ qui sont continues latéralement. Figure $\mathbf{1 0 .}$

Profils en double:

Afin de déterminer le pendage des structures en profondeur, les trainés électriques TE1 et TE2 ont été réalisés avec une double longueur de ligne AB de $200 \mathrm{~m}$ correspondant à une profondeur d'investigation moins élevée (40m).Les figures 12 et 13 montrent la réponse en double de profils TE1 et TE2 :

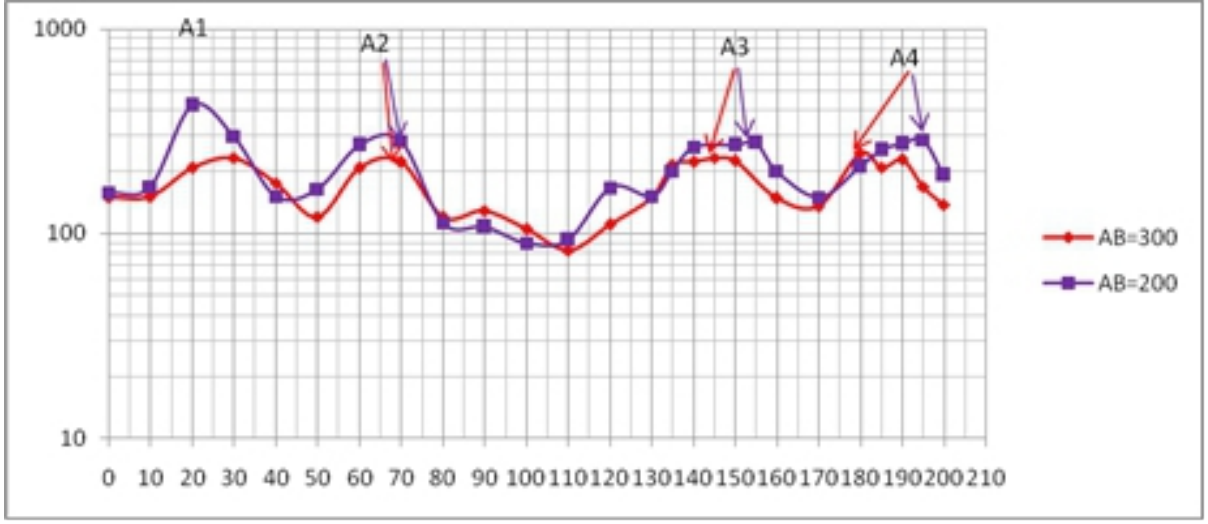

Figure 12 : superposition des profils du traîné électrique TE1.

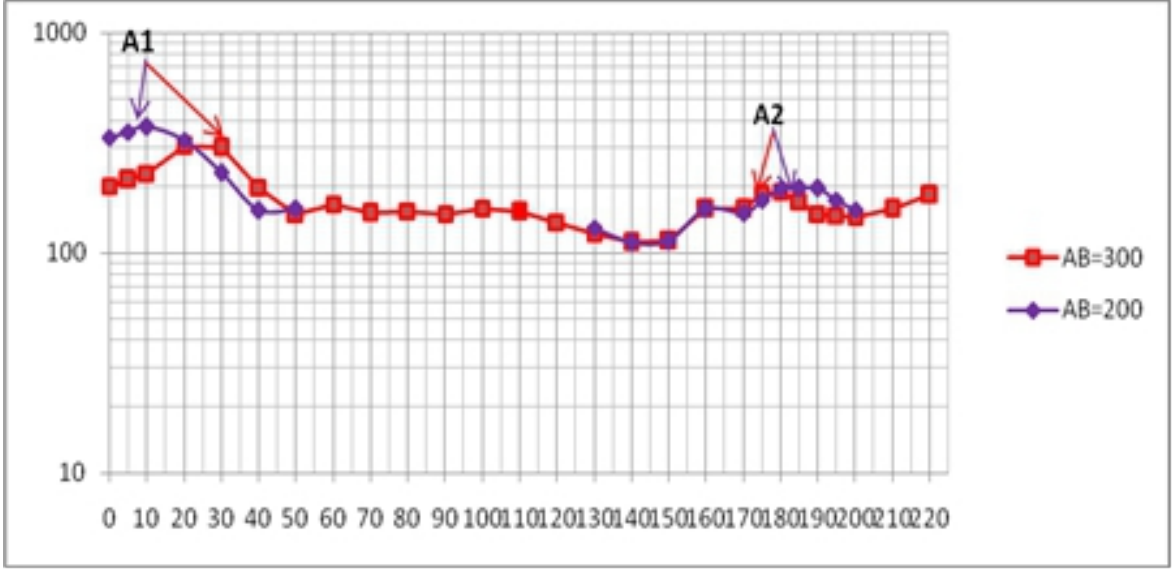

Figure 13 : superposition des profils du TE2.

La comparaison des deux profils de TE1 et de TE2 ne montre pas une coïncidence parfaite de la position des anomalies positives, mais plutôt des décalages dans l'espace de ces anomalies, ceci peut être interprété par l'existence d'un certain pendage des structures minéralisés en profondeur.

\section{Estimation du pendage des structures minéralisées.}

V-3-1.Au niveau du profil TE1.

Le profil de trainé électrique TE1 a mis en évidence quatre anomalies positives A1, A2, A3 et A4 qui présentent un décalage dans l'espace entre 
celui avec un $\mathbf{A B}$ de $\mathbf{2 0 0 m}$ et celui avec un $\mathbf{A B}$ de $\mathbf{3 0 0 m}$.

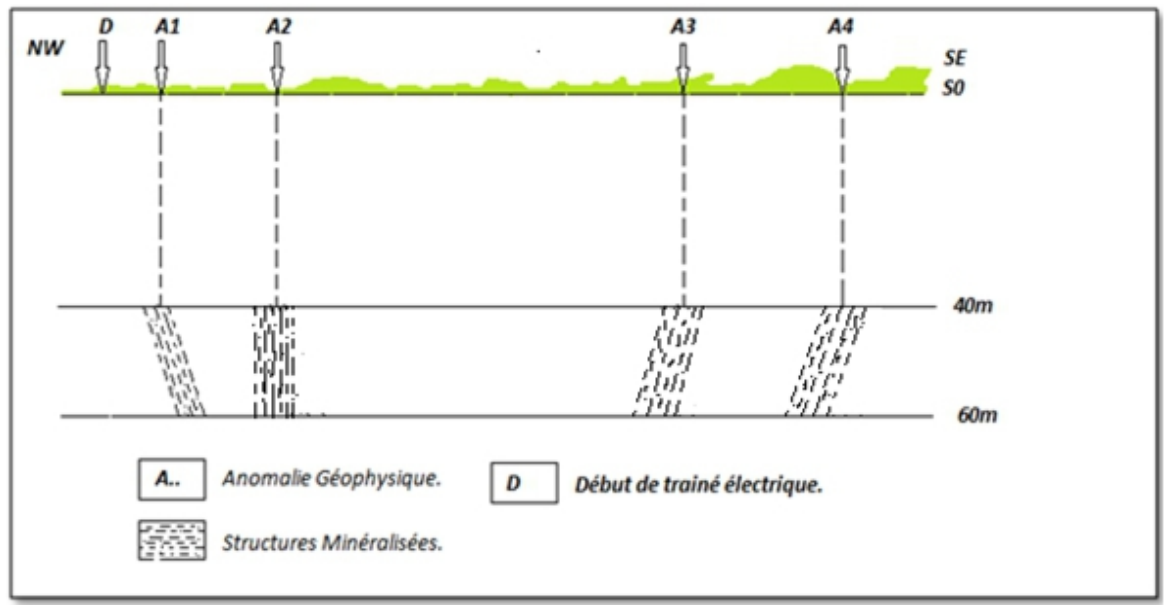

Figure 14 : coupe schématique illustrant le pendage des structures minéralisées de TE1.

\section{Au niveau de l'anomalie A1:}

Entre les deux profils de TE1, l'anomalie A1 présente un décalage de l'ordre de $\mathbf{1 0} \mathbf{~ m}$ vers le SE.

Le pendage qui présente la structure $\mathbf{Z 1}$ est d'ordre de $\mathbf{6 3}^{\circ}$ vers le $\mathbf{S E}$. Au niveau de l'anomalie A2 :

Selon les profils TE1 et TE2, la position de l'anomalie A2 coïncide dans l'espace, ce qui montre que la structure $\mathbf{J}$ est subverticale.

Au niveau de l'anomalie A3 :

L'anomalie $\mathbf{A 3}$ présente un décalage dans l'espace de l'ordre de $\mathbf{1 0} \mathbf{~ m}$ aussi vers le $\mathbf{N W}$ entre $\mathbf{4 0} \mathbf{m}$ et $\mathbf{6 0} \mathbf{m}$ de profondeur.

Le pendage estimé pour la structure $\mathbf{I}$ est de l'ordre de $\mathbf{7 0}^{\circ}$ vers le NW. Au niveau de l'anomalie A4 :

L'anomalie A4 présente un décalage d'ordre $\mathbf{1 5} \mathbf{~ m}$ aussi vers le NW entre $40 \mathrm{~m}$ et $60 \mathrm{~m}$ de profondeur.

La structure $\mathbf{Z 2}$ présente un pendage de $5^{\circ} \mathrm{NW}$.

V-3-2 .Au niveau des profils de TE2:

Les profils de trainé électrique TE2 réalisés au niveau de la zone ou les structures minéralisées sont masquées, ont mis en évidence deux anomalies positives A1et A2, présentant aussi un décalage dans l'espace. 


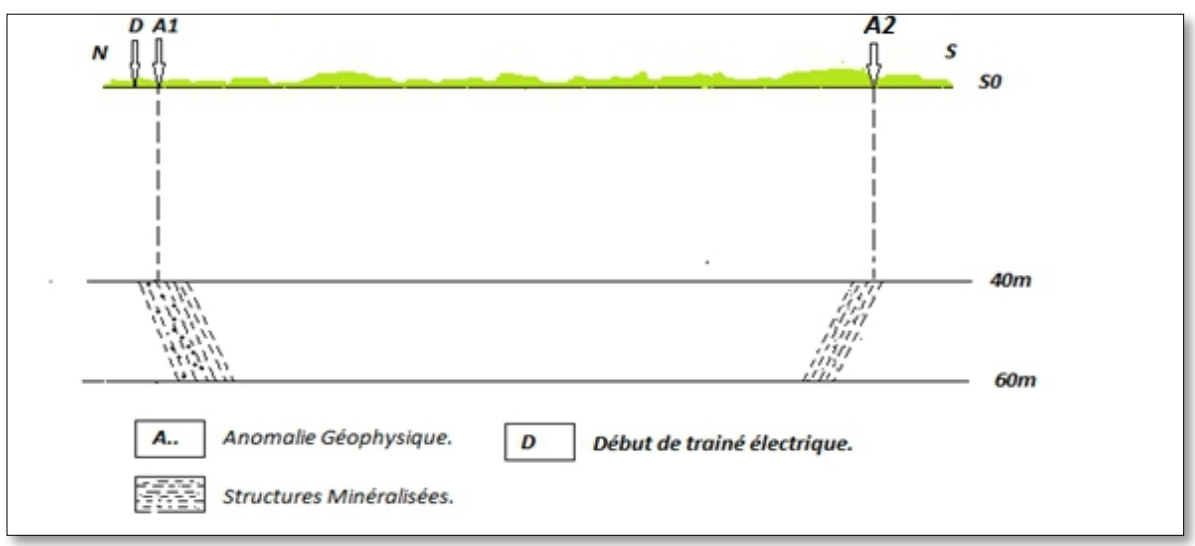

Figure 15 : coupe schématique illustrant le pendage des structures minéralisées de TE2..

\section{Au niveau de l'anomalie A1}

L'anomalie A1 présente un décalage de $\mathbf{1 0 m}$ vers le $\mathbf{N}$ entre les profils de trainé électrique TE2 $(\mathbf{A B}=200$ et $\mathbf{A B}=\mathbf{3 0 0 m})$.

Le pendage que présente la structure $\mathbf{J}$ est de l'ordre de $\mathbf{6 3}^{\circ}$ vers le $\mathbf{S}$.

\section{Au niveau de l'anomalie A2}

L'anomalie A2 correspond à la continuité de la structure $\mathbf{I}$; cette anomalie présente un décalage de $\mathbf{1 5 m}$ mais cette fois ci vers le $\mathbf{N}$ entre $\mathbf{4 0 m}$ et $60 \mathrm{~m}$ de profondeur. Le changement du sens du pendage de cette structure peut être dû fort probablement à l'existence d'un pli synclinal.

Le pendage que présente la structure I est de l'ordre de $\mathbf{5 3}^{\circ}$ mais cette fois-ci vers le $\mathbf{N}$ et en conformité avec le résultat obtenu par le profil TE1 à l'Ouest du secteur.

\section{Conclusion}

Les profils de trainé électrique effectué dans le secteur d'Achemmache permettent de suivre la continuité dans l'espace des structures minéralisées en Tourmaline ainsi que d'estimer leurs pendages. Ils permettent ainsi de :

- montrer la continuité, des structures I et J vers l'Est du secteur; par contre les deux autres structures $\mathbf{Z 1}$ et $\mathbf{Z 2}$ semblent disparaître vers l'Est.

- Monter que les structures I et $\mathbf{Z 2}$ ont un pendage respectivement de $\mathbf{6 3}^{\circ}$ et $53^{\circ} \mathrm{NW}$ alors que la structure $\mathbf{Z 1}$ présente un pendage de $63^{\circ} \mathrm{SE}$. La structure $\mathbf{J}$ est par contre subverticale à l'Ouest et présente un pendage de $63^{\circ} \mathbf{S}$ à l'Est du secteur.

\section{Résultat de la tomographie électrique.}

Dans le secteur d'étude on a réalisé un profil de tomographie électrique TM1 d'une longueur de $\mathbf{2 3 5} \mathbf{~ m}$, afin de comparer les résultats de la 
tomographie avec ceux de la méthode de traîné électrique. Ce profil TM1 a été réalisé à l'endroit du profil de trainé électrique TE1.

L'objectif de cette investigation par tomographie est de confirmer le comportement résistant des structures minéralisées en tourmaline et de suivre leur prolongement en profondeur.

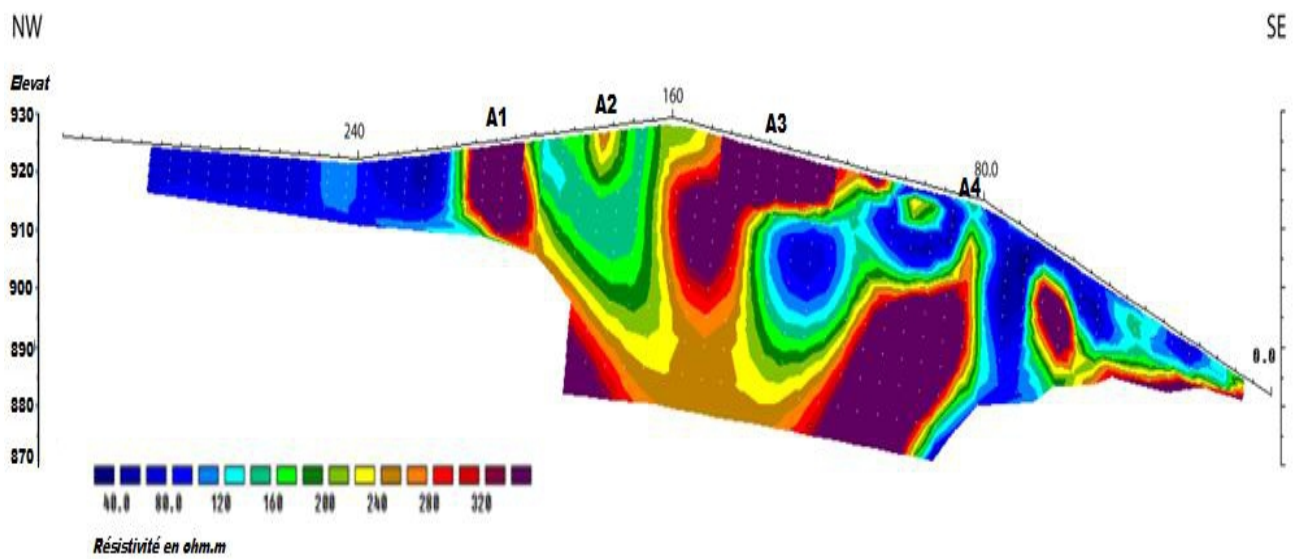

Figure 16 : Profil TM1 de tomographie électrique traversant les structures minéralisées dans le secteur Ouest de la zone d'étude.

\section{Interprétation des résultats de la tomographie électrique.}

Ce profil TM1 montre du NW vers le SE une alternance de niveaux résistants (>200 ohm.m) et de niveaux relativement conducteurs $(<\mathbf{1 2 0}$ ohm.m). Les niveaux résistants sont corrélés aux structures de tourmaline. Les quatre anomalies résistantes correspondant aux structures minéralisées détectées par le profil de trainé électrique TE1 sont confirmés par ce profil de tomographie électrique TM1 et sont :

$>\quad$ La structure $\mathbf{A 1}$ qui se situe dans le profil de tomographie à la distance $\mathbf{X}=\mathbf{2 0 5} \mathrm{m}$ et correspond à la structure $\mathbf{Z 1}$.

$>\quad$ La structure $\mathbf{A 2}$ qui correspond à la structure $\mathbf{J}$ et qui se situe à $\mathbf{X}=\mathbf{1 7 6}$

$\mathrm{m}$.

$>\quad$ La structure $\mathbf{A 3}$ qui correspond à la structure $\mathbf{I}$ et qui se situe à $\mathbf{X}=\mathbf{1 3 0}$ $\mathrm{m}$.

La structure $\mathrm{A4}$ qui correspond à la structure $\mathrm{Z2}$ et qui se situe à $\mathrm{X=80}$ m.

Les résultats de la tomographie corroborent les résultats obtenus par les profils de traîné électrique en double TE1 quand aux pendages des structures minéralisées. 


\section{Discussion et Conclusion}

La zone de cisaillement d'El Hammam constitue le prolongement vers le NE de couloir de cisaillement de Oulmes (Tahiri,1991).ce couloir se subdivise en deux branches, à l'Ouest de la mine d'El Hammam c'est la branche occidentale; à l'Est de celle-ci c'est la branche orientale.

La présente étude a été réalisée au niveau de la branche orientale qui est représentée par des cisaillements décrochants d'orientation NE-SW, et dont les accidents les plus connus sont la faille de Ain El Hammam, la faille des Jebala et la faille d'Achemmèche.

Pour essayer de comprendre les réponses géophysiques obtenues par les mesures réalisées au niveau de la zone d'étude (voir chapitre I), on a représenté les profils des trainés électriques et de tomographie électrique sur la carte structurale de la zone établie par Rahho en 1996 (fig 17).

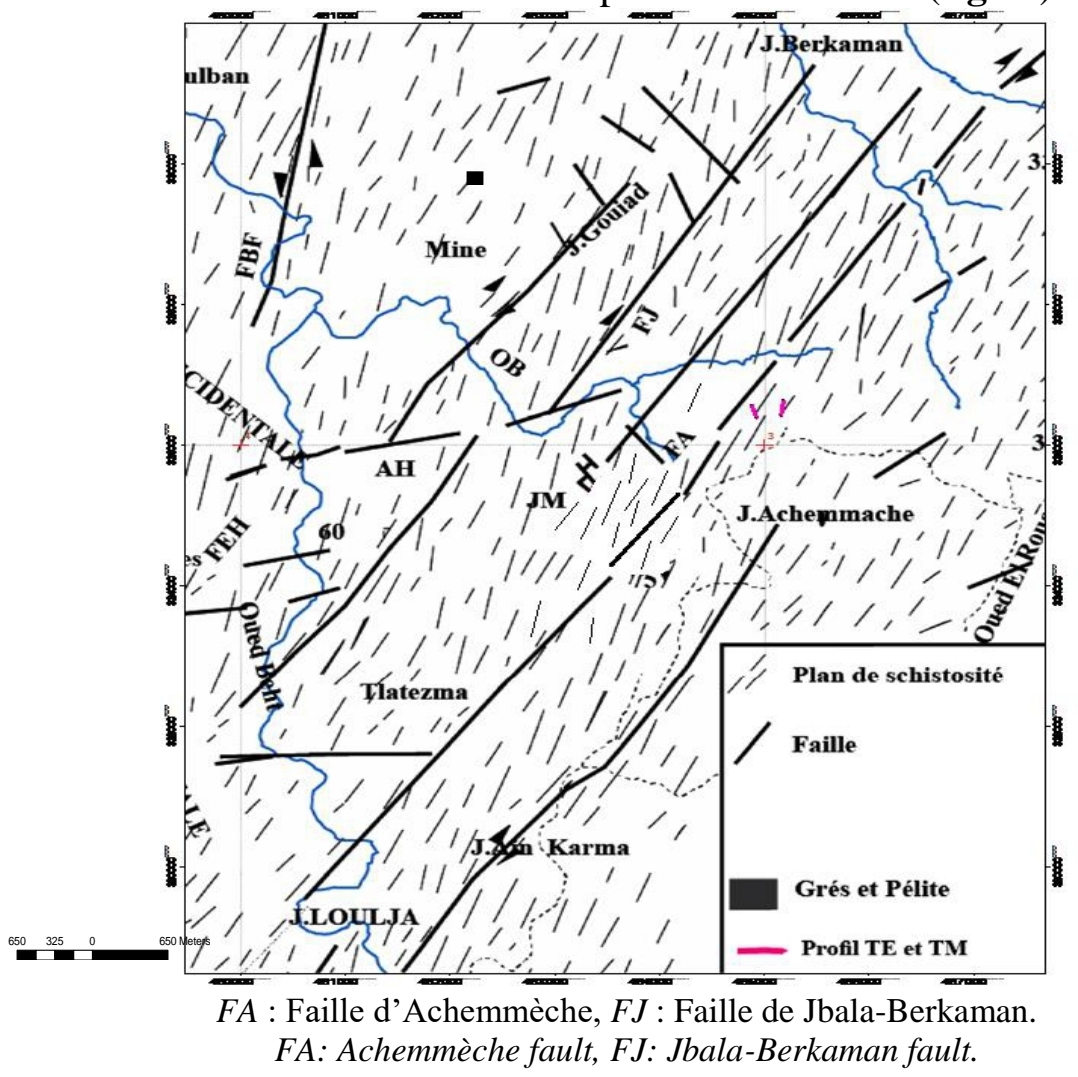

Figure 17: carte structurale de la zone d'étude (Rahho 1996, modifiée).

Sur la figure 17, les profils géophysique réalisés se situent à l'Est de la faille d'Achemmèche (FA), mais non loin de celle-ci.

Les profils géophysiques réalisés par méthodes électrique et tomographie, selon une direction NE-SW, ont mis en évidence $\mathbf{4}$ structures minéralisées A1, A2, A3 et A4 (fig 16), de pendages variables et opposés. . 


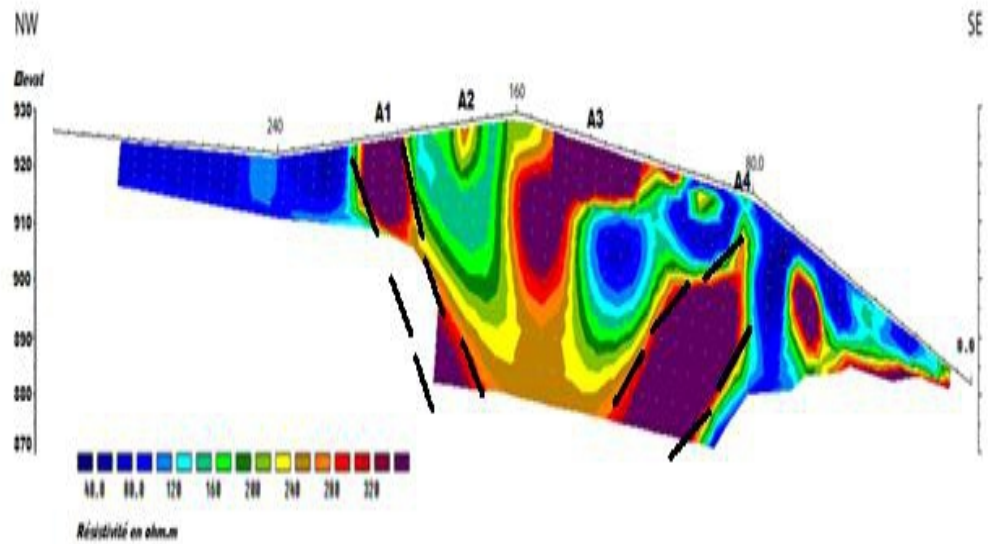

Figure 18 : profil tomographique montrant l'aspect des cisaillements conjugués.

Ces quatre anomalies positives détectées par les essais géophysiques, qui correspondent aux structures minéralisées de tourmaline (voir fig.17), peuvent être considérées comme le remplissage, par tourmaline, quartz et minéraux associés, de failles satellites liées aux jeux de la grande faille d'Achemmèche $(\mathbf{F A})$ et qui pourrait correspondre à une zone nourricière en terme de fluides minéralisateurs.

Les orientations opposées, des structures A1 et A4, peuvent être expliquées par le développement d'un système de failles conjuguées développé lors des déformations de La phase majeure hercynienne qui a engendré des plis hectométriques à kilométriques dont la plupart sont érodés, mais aussi des plis métriques dont certains sont affectés par des cisaillements de type failles inverses (failles NW) et formant des failles conjuguées avec le système de failles d'orientation NE, comme cela a été signalé par Rahho, 1996 (Fig. 19).

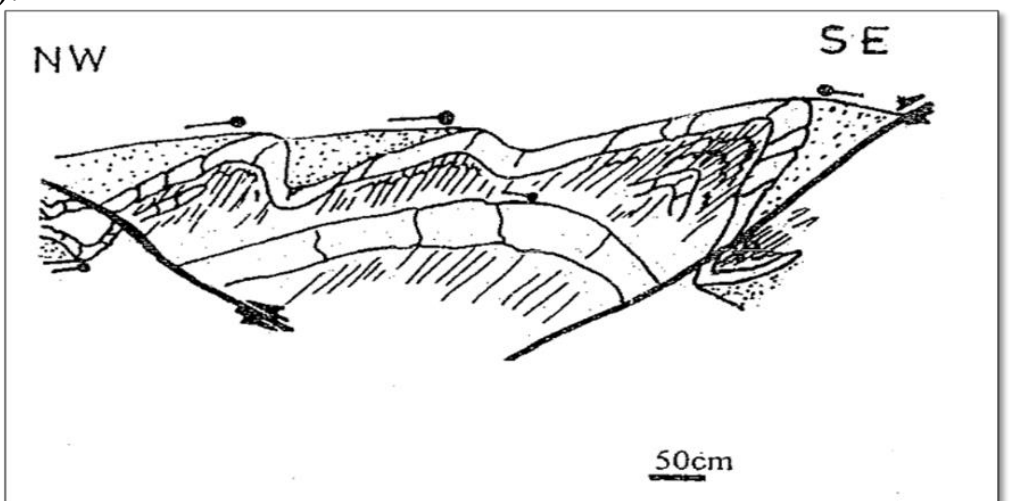

Figure 19 : coupe schématique montrant les cisaillements de type inverse (Rahho, 1996). 


\section{Remerciements}

Les évaluateurs anonymes sont remerciés pour leurs commentaires et suggestions.

\section{References:}

1. ABESSOLO ABENELANG A., BENALI OULHAJ S. HUSSO. Y. 1980- Tectonique et minéralisation dans le champ filonien a Spathfluor d'El Hammam Marcel: Filon principal et filon P3. Stage de 4 éme année. Ecole des Mines de Paris. 32 p.

2. AGAR.J. Données nouvelle sur le district fluorifére d'El hammamBerkamen (Maroc central).

3. AGARD J. 1980- Fluorite, in Géologie des gîtes minéraux marocai n. 2 edit.l. Notes et Mérn. Serv. Géo l. Maroc n.276.

4. AGARD J., BALCON J .M.ORIN PH.1958, Etude géologique et métallogénique de la région minéralisée du Jbel Aouam (Maroc Central). Notes et Mém. Serv. Géol. Maroc, 132.126 p.

5. ALLARY A. LA VENU A., RIBEYROLLES A.1976, Etude tectoniq11e et micro- tectonique d'un segment de chaine hercynienne dans la partie sud orientale du Maroc Central. Notes et Mém. Sen: .Géol., $n \bullet 261,169 p$.

6. EL MAGHRAOUI L.1970, Le gisement de fluorine d'El Hammam Maroc Central. Rapport int. BRPM, $n \bullet 439$-42.

7. JEBRAK M. BENYOUB F.1980, Etude thermométrique des inclusions fluides de quelques filons de fluorine du Maroc. Mines, Géologie et Energie. n• 48. pp. 151- 158.

8. KOSAKEVITCH A. 1965, Description minéralogique du filon de fluorine d'El Hammam, Achemeche, Maroc Central. Rapport SEG t. n' 811. Rabat

9. MI LIAN J.F. -1982- Etude de la partie NW du district d'El Hammam D.E.A. Univ. Orléans.

10. MORIN P.1951, Quelques problèmes relatifs aux roches granitiques et microgranitiques et à leurs minéralisations dans le Maroc Central. Notes Serv. Géol. Maroc. t.4. n.83, pp. 163-182

11. PIQUE A.1976, Evolution sédimentaire du Nord-Ouest de la Meseta marocaine au cours du Carbonifère. Les étapes du comblement du bassin.C.R.Acad.Sc.Frt.282,p.957-960.

12. PIQUE A. 1979, Evolution structurale d'un segment de la chaine hercynienne, la Meseta Marocaine Nord- occidentale. Thèse doct. ès Sc. Strasbourg, 253 p. Sei.GeolMem.56.

13. PIQUE A. MICHARD A. 1981, Les zones structurales du Maroc hercynien. Sei.GeQJ.Bull.34,2,pp.135-146.

14. PIQUE A. KHARBOUCH F.1983, Distension intracontinentale et 
volcanisme associé. La Meseta marocaine nord occidentale au Dévonien-Dinantien. Bull. Centres Rech. Explor.-PrQd. E lfAquitaine. 7.1, pp. 377-387.

15. RAHHO H.1996, Etude géologique de paléozoïque d'El Hammam. Analyse de la fracturation et relation avec les structures minéralisées (Maroc central septentrional).

16. ROUTHIER P.1980, Où sont les métaux pour l'avenir? Les provinces métalliques. Essai de métallogénie globale. Mém. BRGM, Fr. n.105, $410 \mathrm{p}$.

17. VAN MARCKE DE LUMMEN. 1979, Contribution à l'étude pétrographiqoe et minéralogique d'un skarn stannifère. El Hammam (Maroc Central). Mém. thèse L'niv. Louvain. 99 p. 